\title{
Prototyping of Singular Value Reconstruction Precoding for Reliable Non-Orthogonal IoT Signals
}

\author{
Tongyang Xu and Izzat Darwazeh \\ Department of Electronic and Electrical Engineering, University College London, London, UK \\ Email: tongyang.xu.11@ucl.ac.uk, i.darwazeh@ucl.ac.uk
}

\begin{abstract}
Massive connectivity is one of the main research directions for beyond 5G. The cellular based narrowband IoT (NB-IoT), enabled by the orthogonal frequency division multiplexing (OFDM) signal, is an important technique. To evolve into the beyond 5G era, non-orthogonal concepts are preferred to re-shape the NB-IoT to provide higher spectral efficiency, wider coverage and lower power consumed services. This work investigates a non-orthogonal waveform in next generation IoT (NG-IoT) scenarios. Previous work has verified the advantages of zero forcing $(\mathrm{ZF})$ precoding in interference mitigation but with some limitations. This work proposes a singular value reconstruction (SVR) precoding method, which can improve the precoding reliability and greatly reduce noise sensitivity. Simulations show significant spectral efficiency gain when compared with the previous work. An experiment platform is then configured in an over-the-air multiuser multiple input multiple output (MIMO) scenario to verify the practical feasibility of the precoding algorithm.

Index Terms-Prototyping, precoding, multiuser, MIMO, nonorthogonal, IoT, NB-IoT, waveform, reliable communications, software defined radio.
\end{abstract}

\section{INTRODUCTION}

Internet of Things (IoT) is evolving into the next stage, which would employ new signal waveforms to boost spectral efficiency. Existing IoT standard such as narrowband IoT (NB-IoT) [1] applies a narrowband signal format, which occupies 12 sub-carriers over $180 \mathrm{kHz}$ bandwidth. The multicarrier orthogonal frequency division multiplexing (OFDM) signal is the basic waveform and its orthogonality feature simplifies signal recovery. However, for future IoT signals, non-orthogonal signal waveforms would be the direction due to high spectral efficiency and therefore additional high-value services. Non-orthogonal waveforms include a few potential candidates such as filterbank based multicarrier (FBMC) [2], generalized frequency division multiplexing (GFDM) [3], universal-filtered multi-carrier (UFMC) [4] and spectrally efficient frequency division multiplexing (SEFDM) [5], [6].

Taking into account the IoT communication scenarios, signal should be generated with minimal complexity for the purpose of power saving. Therefore, the bandwidth saving technique SEFDM is the focus in this work since it has simpler signal generation when compared with other non-orthogonal signals. In addition to the straightforward signal generation, SEFDM allows for dynamic selection of best parameters to suit a variable radio propagation environment and therefore improves the reliability of communications. The application can potentially double the number of connected devices in restricted areas; extend the signal coverage without consuming extra power and support deeper indoor communications such as in basements where signals are weak. Such advantages are not achievable with existing IoT waveforms.

The benefits achieved in SEFDM are at the cost of extra receiver side computational complexity due to the self-created inter carrier interference (ICI). Sophisticated signal detectors have to be used at the receiver to mitigate the interference. The energy available to each IoT device is limited and any complexity-driven techniques would shorten battery life. One solution is to use the SEFDM waveform technique in the uplink channels, since all the receiver side signal processing will be within a base station (BS), which is not sensitive to power consumption. In order to solve the aforementioned challenges in downlink channels and meet the requirements of future IoT-driven applications, a variety of techniques are being investigated. Precoding is an efficient technique, which can modify signals at the transmitter side and simplify receiver side signal processing. Existing precoding techniques such as dirty paper coding (DPC) [7], vector perturbation (VP) [8], Tomlinson-Harashima precoding (THP) [9] and constructive interference (CI) precoding [10], [11] can significantly improve the performance at the cost of complexity. Linear precoding schemes such as zero forcing (ZF) precoding, simply based on a matrix inversion, shows much lower complexity. Work in [11], [12] have practically tested the ZF precoding method and achieved an improved data rate. However, the ZF precoding has limitations in reliability and the performance degradation would be significant in high noise and high ICI conditions.

This work proposes an alternative precoding methodology via modifying the singular values of a precoding matrix, which will be able to optimize the ill-conditioned ZF precoding matrix [13] and therefore reduce the sensitivity of noise. Since SEFDM ICI characteristics are deterministic, then a pilot based training method is applied to find the optimal singular value combinations for the precoding matrix. To maintain the same complexity as the typical ZF precoding, a look-up table (LUT) is derived for the singular value reconstruction for each specific signal. Simulations are first conducted to show the performance gain of the singular value reconstruction (SVR) precoding. Furthermore, a practical experiment platform is 
configured to validate the proposed precoding method.

\section{BANDWIDTH SAVING WAVEFORM}

The general idea of the bandwidth saving waveform is illustrated in Fig. 1, in which the SEFDM signal shows a compressed bandwidth compared with the OFDM signal when $N=12$ sub-carriers are allocated to both systems. It should be noted that both systems have the same data rate although the SEFDM signal bandwidth is narrower than that of OFDM. Therefore, maintaining the same date rate, the bandwidth compressed SEFDM achieves higher spectral efficiency than OFDM.

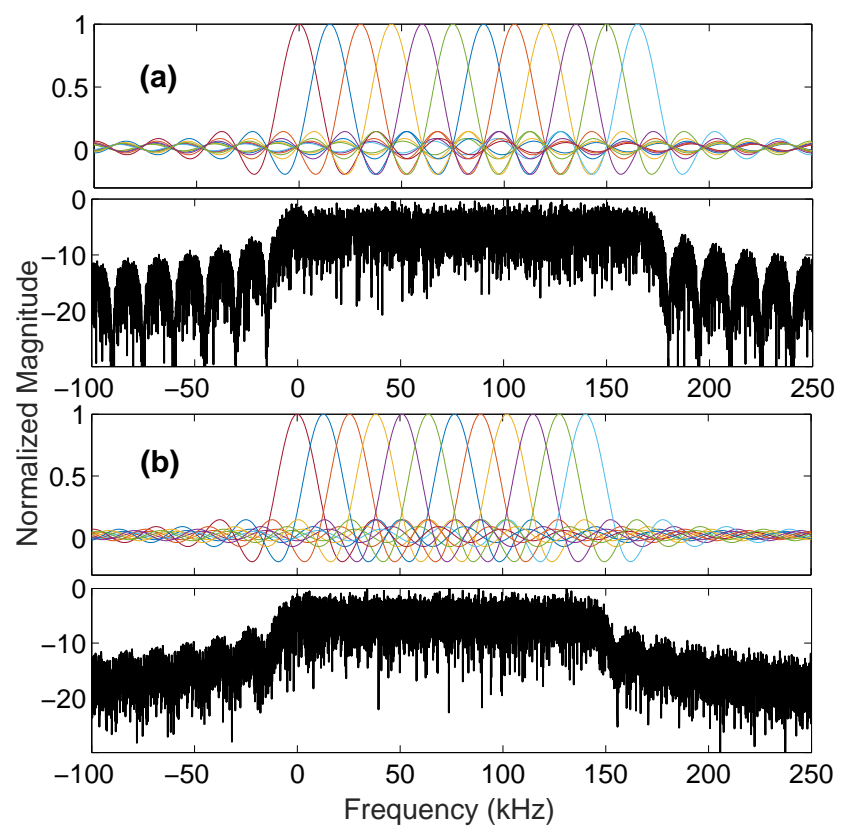

Fig. 1. Spectral illustration for different multicarrier signals. (a) OFDM (12 sub-carriers, bandwidth is $B$ ). (b) SEFDM (12 subcarriers, bandwidth compression factor $\alpha=0.85$, bandwidth is $\alpha \times B$ ).

The simplified mathematical expression of an $N$ sub-carrier SEFDM signal waveform in matrix format is defined as the following

$$
Y=\mathbf{F} S+Z,
$$

where $S$ is an $N$-dimension vector consisting of QPSK symbols, $Z$ is an $N$-dimension vector containing additive white Gaussian noise (AWGN) samples and $\mathbf{F}$ is the $N \times N$ SEFDM modulation matrix defined as $\mathbf{F}=\exp (j 2 \pi n k \alpha / N)$. $n$ is the sub-carrier index ranging from 0 to $N-1, k$ is the time sample index ranging from 0 to $N-1$ and $\alpha=\Delta f \cdot T$ is the bandwidth compression factor. When $\alpha<1$, signal bandwidth is compressed.

Multiplying (1) by a fractional Fourier transform (FrFT) matrix $\mathbf{F}^{*}=\exp (-j 2 \pi n k \alpha / N)$ leading to a demodulated SEFDM signal as

$$
R=\mathbf{F}^{*}(\mathbf{F} S+Z)=\mathbf{C} S+\mathbf{F}^{*} Z=\mathbf{C} S+Z_{\mathbf{F}^{*}},
$$

where $\mathbf{C}$ is the sub-carrier correlation matrix consisting of elements $c(m, n)$. Due to the fractional parameter $\alpha$, ICI [13] exists between the $m^{\text {th }}$ sub-carrier and the $n^{\text {th }}$ sub-carrier given as

$$
\begin{aligned}
& c(m, n)=\frac{1-\exp (j 2 \pi(m-n) \alpha)}{N\left(1-\exp \left(\frac{j 2 \pi(m-n) \alpha}{N}\right)\right)}, \\
& \Re\{c(m, n)\} \neq 0 \quad(\alpha<1, m \neq n), \\
& \Im\{c(m, n)\} \neq 0 \quad(\alpha<1, m \neq n),
\end{aligned}
$$

The self-created ICI is defined when $m \neq n$. Both the real and imaginary parts of $c(m, n)$ are presented in (4) and (5), respectively. It is observed that when $\alpha<1$, both real and imaginary results are non-zeros indicating interference in both parts. To remove efficiently the interference, precoding has to be used at the transmitter.

\section{Precoding Methodology}

ZF precoding is the straightforward and widely used approach in practical communication systems due to its simple signal processing. Thus, the key principle of the ZF precoding is to pre-equalize the symbol $S$ by multiplying with the inverse of the matrix $\mathbf{C}$ before SEFDM modulation. Therefore, the precoded signal $\bar{S}$ is defined as

$$
\bar{S}=\mathbf{C}_{\mathbf{p}} S .
$$

where the precoding matrix $\mathbf{C}_{\mathbf{p}}$ is given as

$$
\mathbf{C}_{\mathbf{p}}=\mathbf{C}^{H}\left(\mathbf{C C}^{H}\right)^{-1} \text {. }
$$

The precoded signal $\bar{S}$ will go through the same operations in (1) and (2). The drawback of this algorithm is in its performance sensitivity in high noise environments due to the unequal singular values of the correlation matrix $\mathbf{C}$ shown in Fig. 2. The minimum singular value results in an illconditioning precoding matrix and therefore greatly compromises the signal-to-noise ratio (SNR).

\section{A. Reconstruction of Singular Values}

Increasing the minimum singular value would result in lower values of the correlation matrix inverse and therefore improve the SNR and SEFDM signal quality. However, the singular value can not be increased extensively so that the use of a precoding matrix would result in inaccurate reproduction of the data at the receiver. Therefore, searching for an optimal singular value is the motivation of this work. Unfortunately, There is no closed form solution to determine such an optimal value in SEFDM. In this work, based on the fact that for a given system specification the correlation matrix C is deterministic, we apply a pilot training methodology to modify intelligently the singular value to optimal (based on minimizing bit error rate (BER)) and therefore reduce the sensitivity of noise effects.

To effect the above, at the start of a data stream, pilot symbols are sent through the system to estimate the BER. The typical ZF precoding BER performance is computed for 


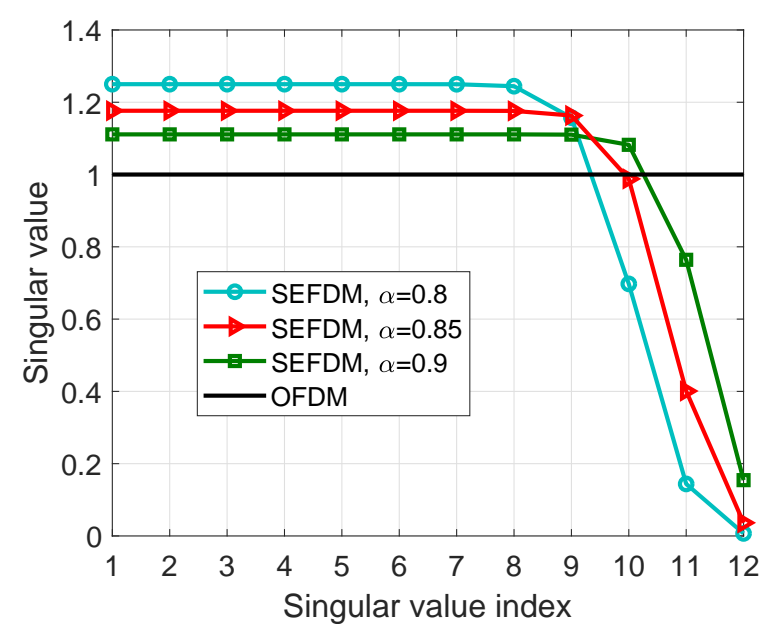

Fig. 2. Singular values of the correlation matrix $\mathbf{C}$ versus singular value index. $\mathrm{N}=12$ sub-carriers are evaluated.

SEFDM as a reference. By tuning the singular values of the ZF precoding matrix in each pilot training, several SVR precoding BER results are obtained. The tuned singular value, associated with the lowest BER values (maximum deviation relative to the reference BER), is the optimum solution. The singular value decomposition (SVD) algorithm [14] is applied to the correlation matrix $\mathbf{C}$ to obtain singular values.

$$
\mathbf{C}=\mathbf{U} \boldsymbol{\Sigma} \mathbf{V}^{*}
$$

where $\mathbf{U}$ and $\mathbf{V}^{*}$ are unitary matrices and $\boldsymbol{\Sigma}$ is a diagonal matrix that contains the singular values $\operatorname{diagonal}(\boldsymbol{\Sigma})=$ $\left[\sigma_{0}, \sigma_{1}, \ldots, \sigma_{N-1}\right]$ of $\mathbf{C}$.

We maintain all other singular values while changing the singular value $\sigma_{N-1}$ during each pilot training. Each singular value modification requires one BER calculation, which is later compared with the reference BER. It should be noted that the singular value tuning range is limited to $\left[\sigma_{N-1}, \sigma_{N-2}\right]$, where $\sigma_{N-1}$ is the minimum singular value and $\sigma_{N-2}$ is the second minimum singular value. The singular value update starts from $\sigma_{N-1}$ to $\sigma_{N-2}$ with a step size of $\sigma_{s t e p}=0.001$. After the pilot training, the tuned singular value, $\tilde{\sigma}_{N-1}$ leading to the best BER performance is selected as the optimal one to construct a modified precoding matrix as

$$
\tilde{\mathbf{C}}_{\mathbf{p}}=\mathbf{V} \tilde{\boldsymbol{\Sigma}}^{-1} \mathbf{U}^{*}
$$

where $\operatorname{diagonal}\left(\tilde{\boldsymbol{\Sigma}}^{-1}\right)=\left[1 / \sigma_{0}, 1 / \sigma_{1}, \ldots, 1 / \tilde{\sigma}_{N-1}\right]$ are the reconstructed inverse singular values.

Table I presents the reconstructed singular value look-up table. It shows that when the singular value is configured to be 0.207 , the system with $10 \%$ bandwidth compression would achieve the optimal performance. While for the $15 \%$ bandwidth compression, the optimal singular value should be 0.063 . Finally the optimal singular value for the $20 \%$ bandwidth compression is 0.020 . The derived singular values in Table I will be used in the following simulation and experiment validation.

Table I: Singular value reconstruction look-up table

\begin{tabular}{|l|l|l|}
\hline$\alpha$ & Original $\sigma_{\min }$ & Modified $\tilde{\sigma}_{\min }$ \\
\hline \hline 0.9 & 0.129 & 0.207 \\
\hline 0.85 & 0.027 & 0.063 \\
\hline 0.8 & 0.005 & 0.020 \\
\hline
\end{tabular}

In precoded communication systems, the quality of a precoding matrix is defined by the condition number. The condition number of the correlation matrix $\mathbf{C}$ can be defined as

$$
\kappa=\frac{\sigma_{\max }}{\sigma_{\min }}
$$

where $\sigma_{\max }$ indicates the maximum singular value and $\sigma_{\min }$ is the minimum singular value. The condition number varies in different communication systems. Large condition numbers would decay system performance, which is the case in SEFDM systems.

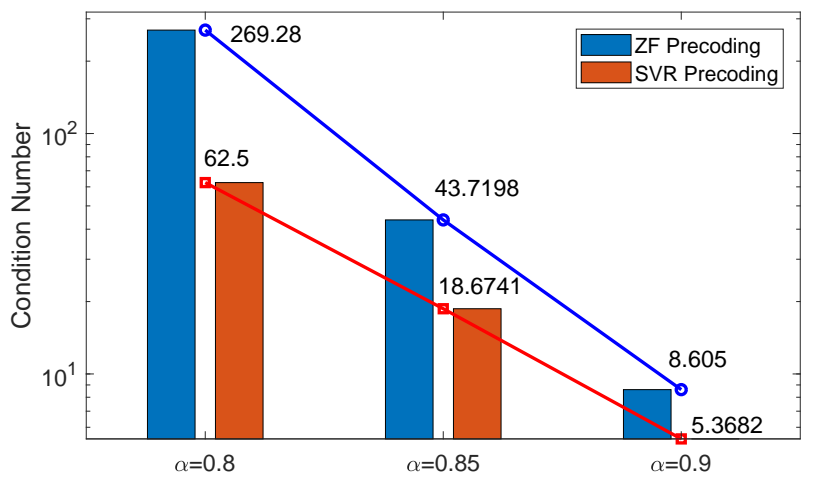

Fig. 3. Condition number improvement via singular value reconstruction.

The condition numbers at different $\alpha$ in SEFDM are presented in Fig. 3, in which the condition number is rapidly decreased with the increase of $\alpha$. This indicates that the higher bandwidth compression results in larger condition numbers and therefore worse system performance. After fine tuning the singular values in SVR precoding, Fig. 3 clearly shows that the condition numbers are greatly reduced for all the bandwidth compression scenarios.

The effect of large condition number is demonstrated in Fig. 4(a) where the data signal is distributed over the entire signal band resulting in a spectrum hole and high out-of-band power leakages. The potential issue of such an ill-conditioned spectral pattern is its high noise sensitivity since a minor noise would bury the data signal. The spectral advantage of modifying the minimum singular value is illustrated in Fig. 4(b). With the proposed singular value reconstruction method, the power of the signal is concentrated to the data spectral band and therefore reduced noise sensitivity. 

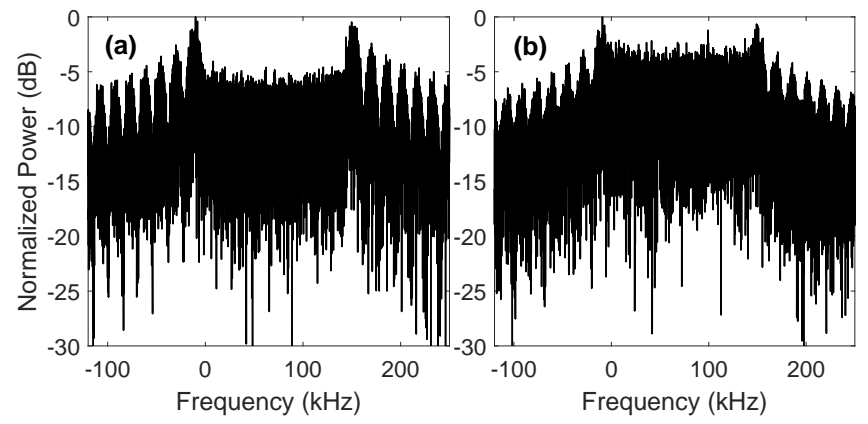

Fig. 4. Spectra comparisons with $15 \%$ bandwidth saving $(\alpha=0.85)$ after two precoding schemes. (a) ZF precoding. (b) SVR precoding.

The computational complexity of precoding is within a base station, which is not sensitive to extra signal processing pressure due to its sufficient power supply. Receiver side, which is an IoT device in our work, follows the traditional signal demodulation methodology. In terms of SEFDM signal demodulation, the work in [5] introduced simplified methods, which have similar complexity when compared with typical OFDM signal demodulation. For the proposed SVR precoding method, a codebook-like look-up strategy is derived in Table I for each specific signal. Therefore, the newly proposed precoding technique has reasonable computational complexity.

\section{Modelling Results}

In IoT communications, signals are simply designed using low order modulation formats and a small number of subcarriers for power saving purpose. IoT devices are normally deployed in stable conditions for many years and the wireless channel is nearly static and no frequency selective fading [15] exists. Therefore, the simulation in this work is operated in an AWGN channel with $N=12$ sub-carriers and low order modulation format QPSK. The signal configurations are following the 3GPP NB-IoT standard [1] except the sub-carrier spacing, which is smaller than the standardized $15 \mathrm{kHz}$ due to non-orthogonal sub-carrier packing. For downlink NB-IoT channels, the convolutional code [1] with coding rate 1/3 [16] is used to improve further the BER performance.

Fig. 5 compares uncoded spectral efficiency of systems with various bandwidth compression factors and different precoding schemes. It is clearly seen that spectral efficiency is significantly improved with the reduction of bandwidth compression factors. At low Eb/N0 range, the SVR precoding outperforms the typical ZF precoding in all the bandwidth compression scenarios. With the increase of $\mathrm{Eb} / \mathrm{N} 0$, the performance of the typical ZF precoding is improved and approaches the SVR precoding results at high $\mathrm{Eb} / \mathrm{N} 0$. When $\alpha=0.9$, both precoding schemes have close performance. However, with the introduction of higher ICI, the gap between the typical ZF precoding method and the proposed SVR precoding method is widened.

Fig. 6 shows results with convolutional coding under different bandwidth compression factors in the two precoding

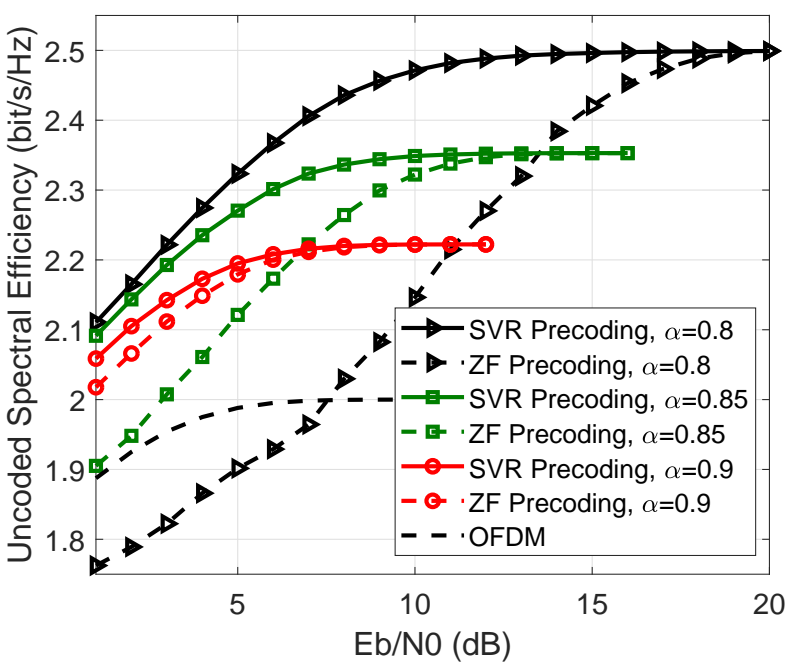

Fig. 5. Uncoded spectral efficiency comparisons.

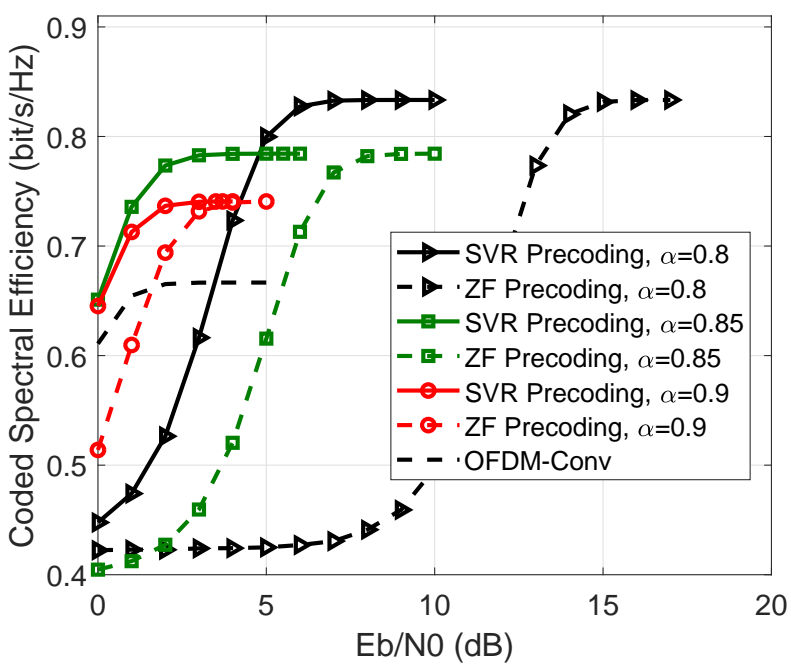

Fig. 6. Coded spectral efficiency comparisons. Coding rate $1 / 3$ convolutional code is considered.

systems. The similar trends for all the systems are observed except the lower spectral efficiency due to the channel coding redundancy. Moreover, with the help of channel coding, all the systems approach the maximum spectral efficiency faster than the uncoded systems. Nevertheless, the proposed SVR precoding technique still outperforms the typical ZF precoding technique in all the bandwidth compression scenarios. It indicates that the singular value reconstruction method is robust in both uncoded and coded scenarios.

\section{EXPERIMENT VALIDATIONS}

The experiment is operated on the NG-IoT platform [12] as illustrated in Fig. 7. To show the proposed idea in a wide range of scenarios, a multi-user multiple input multiple output 


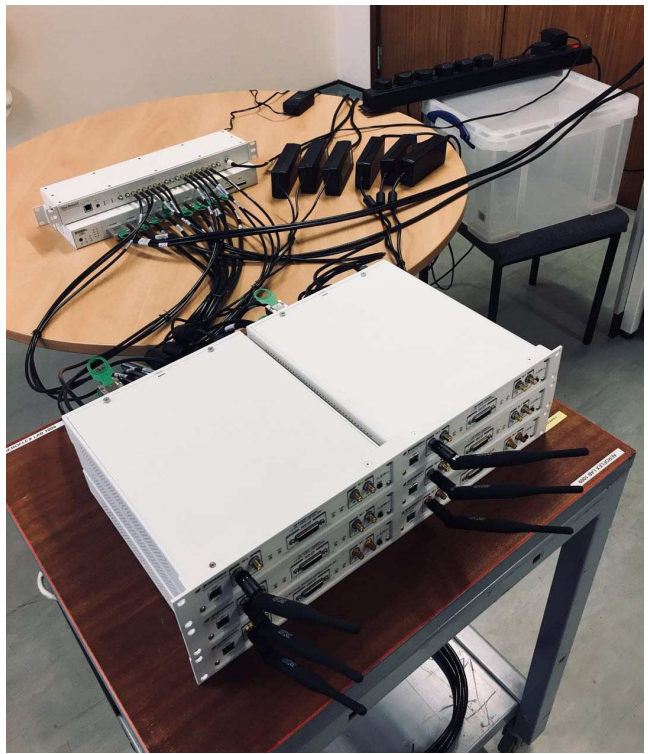

Fig. 7. USRP array setup for the MIMO-SEFDM precoding system.

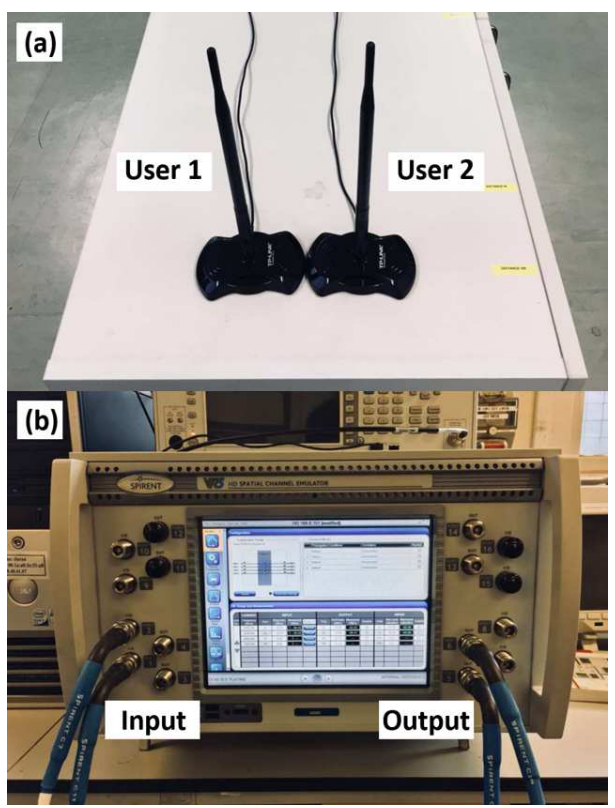

Fig. 8. (a) Receiver side user antennas. (b) Channel emulator VR5 for path loss and noise emulation.

(MIMO) system is applied, which includes six universal software radio peripheral (USRP) transmitter antennas and two receiver side users. The MIMO spatial interference is pre-equalized using the MIMO precoding method in [12]. The interference caused by the non-orthogonally packed subcarriers is solved via the waveform precoding either using the typical ZF precoding or the proposed SVR precoding methods.

The required devices for the experiment are listed in Table II. The experiment shows a real-time $6 \times 2$ multi-user MIMO-SEFDM system for the non-orthogonal IoT communications. Over-the-air MIMO-SEFDM transmission link is realized by using six Omni-directional antennas working at $2.4 \mathrm{GHz}$. Therefore, six National Instruments (NI) USRP$2953 \mathrm{R}$ devices are required to realize the $6 \times 2$ MIMO-SEFDM transmission system. A workstation is used to generate raw digital signals in LabVIEW. The raw digital signal is delivered to a Cabled PCIE Switch Box (x4, 10-port) via a MXI-Express cable (Gen $2 \times 8$, Copper). The Switch Box is very similar to a serial-to-parallel converter, which is used to separate one data stream (from the workstation) to six data streams (to six USRPs). After the separation, six data streams are sent from the Switch Box to six USRPs via six MXI-Express cables (Gen $1 \mathrm{x} 4$, Copper). Then the data stream in each USRP will be digital-to-analogue converted and up converted to the RF frequency at $2.4 \mathrm{GHz}$. The analogue data will be sent out via the Omni-directional antennas over-the-air. The receivers will do the reverse operation to the transmitter side. In order to synchronize six USRPs, a CDA-2990 8 Channel Clock Distribution Accessory module has to be used. This module is used to generate a $10 \mathrm{MHz}$ reference signal and a pulse per second (PPS) signal. The two control signals will be broadcasted to synchronize six separate USRPs via SMA cables.

Table II: Required devices for the NG-IoT platform

\begin{tabular}{|l|l|l|}
\hline Item & Description & Quantity \\
\hline \hline 1 & NI USRP-2953R & 6 \\
\hline 2 & Cabled PCIE Switch Box x4, 10-Port & 1 \\
\hline 3 & MXI-Express cable, Gen 1 x4, Copper & 6 \\
\hline 4 & MXI-Express cable, Gen 2 x8, Copper & 1 \\
\hline 5 & $\begin{array}{l}\text { CDA-2990 8 Channel Clock Distribution } \\
\text { Accessory }\end{array}$ & 1 \\
\hline 6 & Monitor + VGA cable & 1 \\
\hline 7 & BS Omni-directional antennas & 6 \\
\hline 8 & User Omni-directional antennas & 2 \\
\hline 9 & Workstation & 1 \\
\hline
\end{tabular}

The digital signal is configured based on the specifications in Table III and therefore the user side received signal spectrum is demonstrated in Fig. 9. The signal band is faded and not flat after the ZF precoding method in Fig. 9(a) resulting in potential poor performance. This is due to the ill-conditioning of the correlation matrix $\mathbf{C}$. The solution is to intentionally amplify small singular values of the correlation matrix. The proposed SVR precoding signal spectrum is shown in Fig. 9(b), in which the signal band becomes flat indicating the robust performance even in high noise conditions.

The experimental spectral efficiency is computed and compared in Fig. 10, in which both precoding schemes enable higher spectral efficiency than the typical OFDM when SNR reaches a certain level. However, the proposed SVR precoding method achieves significantly better result than the typical ZF precoding method at low SNR range. This practically validates the robust performance of SVR precoding in high noise conditions. It should be noted that the typical ZF precoding based SEFDM system performs even worse than the OFDM in high noise range, which indicates the unreliability of the ZF precoding in extreme noise environments. 
Table III: Experiment signal specifications

\begin{tabular}{lll}
\hline \hline Parameter & OFDM & SEFDM \\
\hline No. of base station antennas & 6 & 6 \\
No. of users & 2 & 2 \\
RF center frequency $(\mathrm{GHz})$ & 2.4 & 2.4 \\
Sampling frequency $(\mathrm{MHz})$ & 1.92 & 1.92 \\
IFFT sample length & 128 & 128 \\
No. of guard band sub-carriers & 58 & 58 \\
No. of data sub-carriers & 12 & 12 \\
No. of cyclic prefix samples & 10 & 10 \\
Sub-carrier bandwidth $(\mathrm{kHz})$ & 15 & 15 \\
Bandwidth compression factor $\alpha$ & 1 & 0.85 \\
Sub-carrier spacing (kHz) & 15 & $15 \times \alpha$ \\
Bandwidth (kHz) & 180 & $180 \times \alpha$ \\
Modulation scheme & QPSK & QPSK \\
Maximum Spectral Efficiency (bit/s/Hz) & 2 & $2 / \alpha$ \\
\hline
\end{tabular}

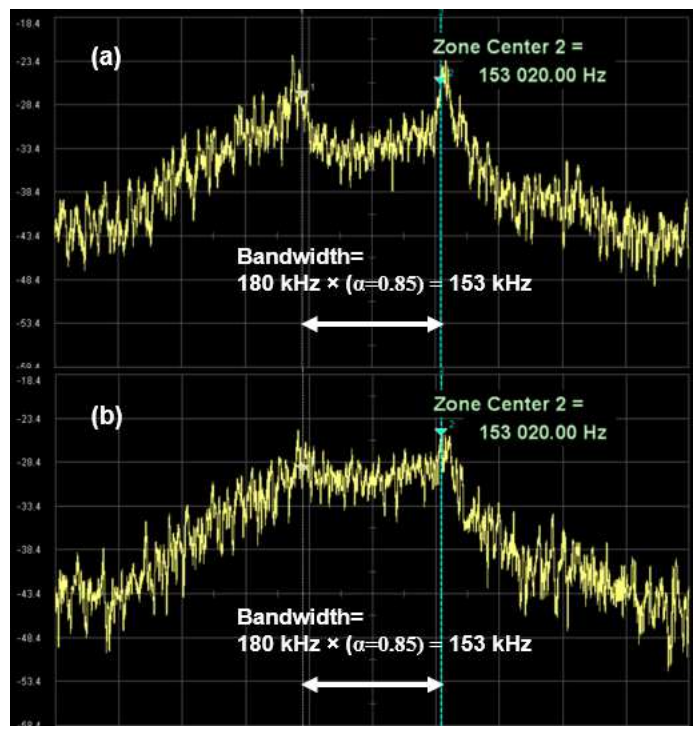

Fig. 9. Spectra comparison. (a) Typical ZF precoding. (b) Proposed SVR precoding.

\section{CONCLUSIONS}

This work focuses on dealing with a realistic challenge in non-orthogonal IoT signal communications. The nonorthogonal concept improves further the IoT signals spectral efficiency by transmitting the same amount of information in a narrower spectral bandwidth. Spectral efficiency is improved at the expense of complex signal detection. To release IoT devices burden, signal precoding is employed at the transmitter to pre-equalize non-orthogonal signals. However, ZF precoding method is not reliable in high ICI and high noise environments. Therefore, this work proposed a reliable precoding scheme to deal with the ill-conditioned problem in non-orthogonal SEFDM signal waveforms via optimizing singular values of the precoding matrix. A pilot based training approach is applied to determine optimal singular values. Both simulation and experiment are designed to verify the noise robustness and faster spectral efficiency gain achievement after using the singular value reconstruction precoding.

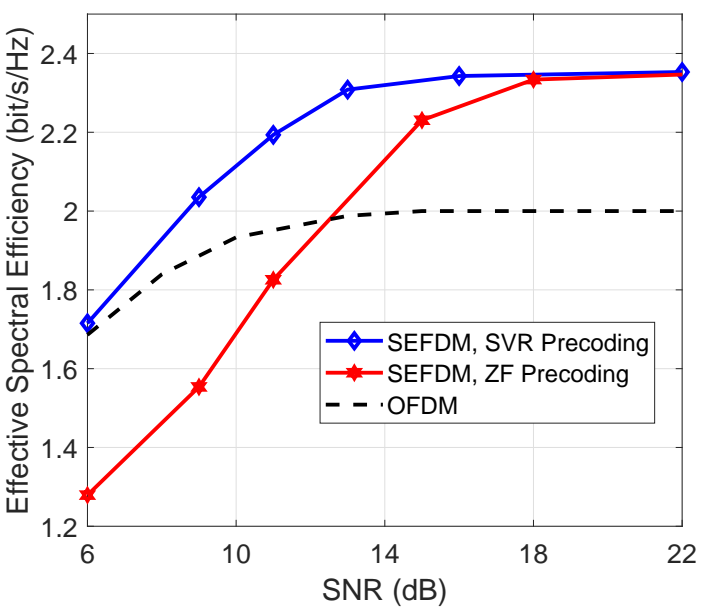

Fig. 10. Effective spectral efficiency comparison for two precoding schemes in the $15 \%$ bandwidth compression scenario $(\alpha=0.85)$.

\section{REFERENCES}

[1] 3GPP TS 36.213 v.14.2.0, "LTE; evolved universal terrestrial radio access (E-UTRA); physical layer procedures," Rel. 14, Apr. 2017.

[2] B. Farhang-Boroujeny, "OFDM versus filter bank multicarrier," IEEE Signal Processing Magazine, vol. 28, no. 3, pp. 92-112, May 2011.

[3] N. Michailow, M. Matthe, I. Gaspar, A. Caldevilla, L. Mendes, A. Festag, and G. Fettweis, "Generalized frequency division multiplexing for 5th generation cellular networks," IEEE Transactions on Communications, vol. 62, no. 9, pp. 3045-3061, Sep. 2014.

[4] V. Vakilian, T. Wild, F. Schaich, S. Ten Brink, and J.-F. Frigon, "Universal-filtered multi-carrier technique for wireless systems beyond LTE," in IEEE Globecom Workshops, Dec. 2013, pp. 223-228.

[5] T. Xu and I. Darwazeh, "Transmission experiment of bandwidth compressed carrier aggregation in a realistic fading channel," IEEE Transactions on Vehicular Technology, vol. 66, no. 5, pp. 4087-4097, May 2017.

[6] H. Ghannam, D. Nopchinda, M. Gavell, H. Zirath, and I. Darwazeh, "Experimental demonstration of spectrally efficient frequency division multiplexing transmissions at E-Band," IEEE Transactions on Microwave Theory and Techniques, vol. 67, no. 5, pp. 1911-1923, 2019.

[7] M. Costa, "Writing on dirty paper (Corresp.)," IEEE Transactions on Information Theory, vol. 29, no. 3, pp. 439-441, May 1983.

[8] J. Maurer, J. Jalden, D. Seethaler, and G. Matz, "Vector perturbation precoding revisited," IEEE Transactions on Signal Processing, vol. 59, no. 1, pp. 315-328, Jan. 2011.

[9] C. Windpassinger, R. F. H. Fischer, T. Vencel, and J. B. Huber, "Precoding in multiantenna and multiuser communications," IEEE Transactions on Wireless Communications, vol. 3, no. 4, pp. 1305-1316, Jul. 2004.

[10] C. Masouros and G. Zheng, "Exploiting known interference as green signal power for downlink beamforming optimization," IEEE Transactions on Signal Processing, vol. 63, no. 14, pp. 3628-3640, Jul. 2015.

[11] T. Xu, F. Liu, A. Li, C. Masouros, and I. Darwazeh, "Constructive interference precoding for reliable non-orthogonal IoT signaling," in IEEE INFOCOM 2019, Apr. 2019, pp. 590-595.

[12] T. Xu, C. Masouros, and I. Darwazeh, "Waveform and space precoding for next generation downlink narrowband IoT," IEEE Internet of Things Journal, vol. 6, no. 3, pp. 5097-5107, Jun. 2019.

[13] S. Isam and I. Darwazeh, "Precoded spectrally efficient FDM system," in 21st IEEE International Symposium on Personal Indoor and Mobile Radio Communications (PIMRC), Sep. 2010, pp. 99-104.

[14] J. Proakis and M. Salehi, Digital Communications. McGraw-Hill, 2008.

[15] G. Ku and J. M. Walsh, "Resource allocation and link adaptation in LTE and LTE-Advanced: A tutorial," IEEE Communications Surveys Tutorials, vol. 17, no. 3, pp. 1605-1633, thirdquarter 2015.

[16] H. Fattah, 5G LTE Narrowband Internet of Things (NB-IoT). CRC Press, 2018. 